The Be Phenomenon in Early-Type Stars, IAU Colloquium 175

ASP Conference Series, Vol. 214, 2000

M. A. Smith, H. F. Henrichs, and J. Fabregat, eds.

\title{
Substantial Disc Loss in the Be/X-ray Binary System A0535+26
}

\section{N.J. Haigh}

Department of Physics and Astronomy, The University, Southampton, SO17 1BJ, UK.

\section{M.J. Coe}

Department of Physics and Astronomy, The University, Southampton, SO17 1BJ, UK.

\section{I.A. Steele}

Astrophysics Reseearch Institute, Liverpool John Moores University, Twelve Quays House, Egerton Wharf, Birkenhead, CH41 1LD, UK.

\section{J. Fabregat}

Departamento de Astronomia y Astrofisica, Universidad de Valencia, E-46100 Burjassot, Valencia, Spain.

\begin{abstract}
.
Infrared $J H K$ photometry from October 1998 revealed a remarkable change in the $\mathrm{Be} / \mathrm{X}$-ray binary system $\mathrm{A} 0535+26$, manifesting itself as a dramatic reduction in emission from the circumstellar disc. Optical and IR spectra presented here have confirmed this disc-loss in greater detail showing radically reduced Hydrogen and Helium line emission, to a degree never before seen in this system, and only rarely in a handful of $\mathrm{Be} / \mathrm{X}$-ray binaries. Optical spectroscopy has revealed the re-appearance of a small, stable inner disc, and IR spectra/photometry show the eventual regrowth of a robust circumstellar disc resembling the pre-disc-loss state.
\end{abstract}

\section{Introduction}

The classical HMXRB system A0535+26, discovered in 1975 (Coe et al. 1975; Rosenberg et al. 1975) manifests itself optically as the O9.7IIIe (Steele et al. 1998) star HDE 245770.

Hayakawa (1981) described a 104s period neutron star in a 110 day orbit. Accretion onto the NS from the Be circumstellar disc near perihelion is believed to give rise to periodic 'normal' outbursts, whilst unpredictable non-perihelic 'giant' outbursts occur more randomly, probably from discrete mass-loss events striking the NS. HDE 245770 displays optically variability on many timescales, probably all attributable to changes in the circumstellar disc. Optical spectra have revealed $\mathrm{H} \alpha$ emission throughout its observed history (Figure 1), though 


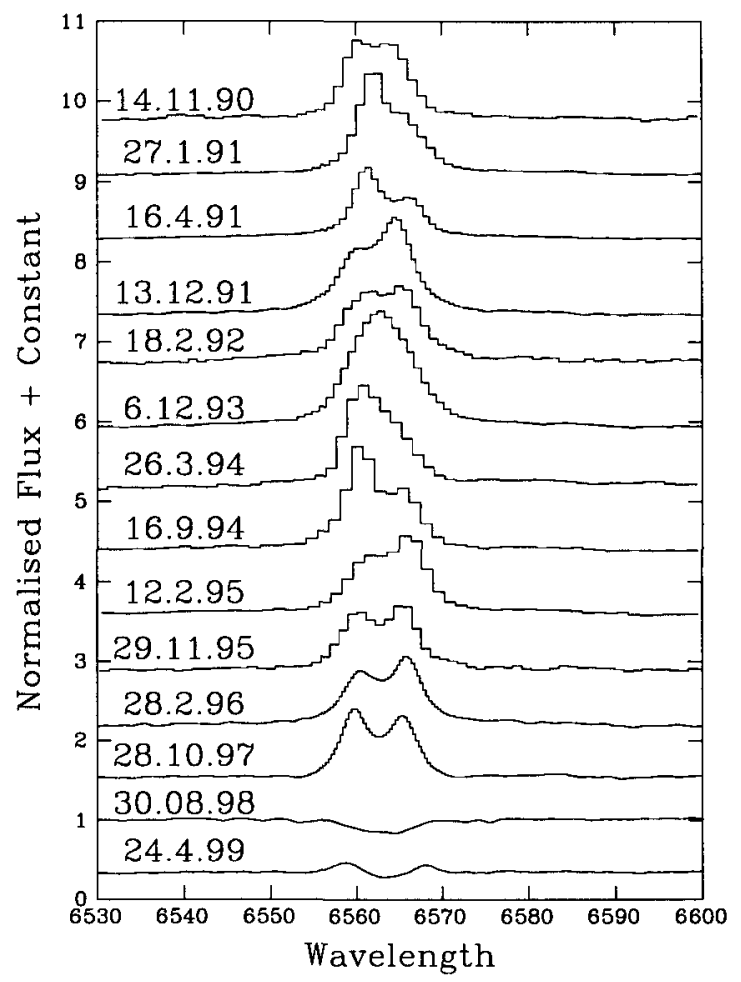

Figure 1. Variation of $\mathrm{H} \alpha$ spectra. The 1990-97 data are from Clark et al. (1998), and 1998-99 are from Haigh et al. (1999).

with varying $\mathrm{EW}$ and profile, frequently being double peaked and displaying evidence for $\mathrm{V} / \mathrm{R}$ cycles of around 1 year.

Here we report on optical/IR observations regarding an episode of probable complete disc-loss, and the reappearance of a new disc.

\section{Observations}

Optical spectra have largely been obtained from the ING service programme; spectroscopy from the Isaac Newton Telescope (INT) and the William Herschel Telescope (WHT). Further spectra have been obtained at the Kitt Peak National Observatory (KPNO) Coudé-Feed Telescope (Douglas Gies, personal communication), and the $1.9 \mathrm{~m}$ at the South African Astrophysical Observatory (SAAO). Optical photometry have come from the $1.0 \mathrm{~m}$ also at SAAO, and the Jacobus Kapteyn Telescope (JKT). ZJHK spectra have been obtained with the CGS4 instrument on the United Kingdom Infra-red Telescope (UKIRT). The Telescopio Carlos Sanchez (TCS) at Teide observatory, Tenerife, has provided JHK photometry over several years. 


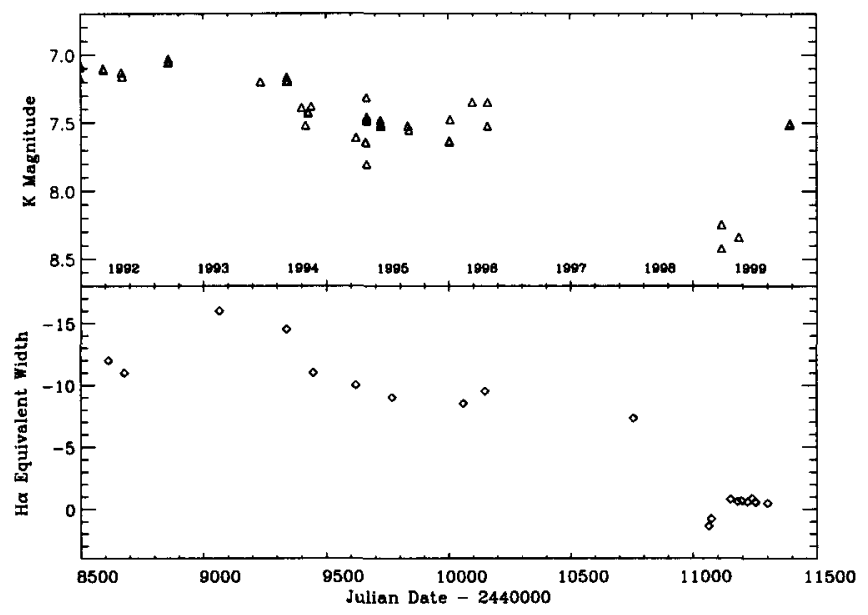

Figure 2. $\quad K$ band photometry from TCS observations and $\mathrm{H} \alpha$ equivalent width data from 1992 to 1999.

\section{Discussion}

The KPNO spectrum from 30 August 1998 (Figure 1) shows $\mathrm{H} \alpha$ in absorption, something not seen in over 20 years of observation of this source. The lower panel of Figure 2 shows a rapid increase in equivalent width (EW) over several weeks after this date, followed by a standstill for at least 7 months; as of 24 April 1999 no further significant change had occurred.

We observed a strong fading in $B V R I J H K$ since previous observations, from $\sim 1$ magnitude in $K$ (Figure 2, top) to $\sim 0.3$ at $B$ and $V$, with associated bluer, lower colour indices. Such photometry confirms an optically faint state; $B V R I J H K$ are all fainter than at any stage since discovery.

$K$ band IR spectra from before and during the disc-loss episode (Figure 3, upper 2 spectra) show $\mathrm{Br} \gamma$ echoing the loss of $\mathrm{H} \alpha$, as expected.

Together these observations (loss of Hydrogen emission, optical/IR fading and IR colour change) provide a strong indicator of circumstellar disc loss. The observed flux decrease matches the predicted disc contribution, as the Be star briefly attempted to become a B star.

As Figure 2 shows, $\mathrm{H} \alpha \mathrm{EW}$ behaviour both before and throughout this episode is beautifully tracked by the $\mathrm{K}$ band magnitude. This clearly demonstrates the origin of both the IR excess and Balmer line emission in similar regions of the system, and shows their equal effectiveness for probing the extent of the disc; indeed it was $\mathrm{K}$ band photometry which first alerted us to the disc-loss.

The assumption of a predominantly kinematic explanation of the line profile, combined with the overall low IR luminosity, implies the existence from approximately November 1998 until at least April 1999 of a rapidly rotating and thus small inner disc. Such a small radius is to be expected of a young disc which has had insufficient time to propagate material outwards. This emerging 


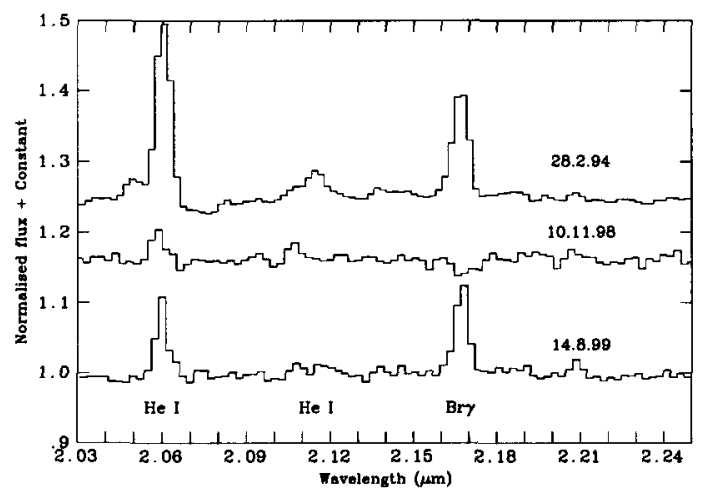

Figure 3. CGS4 K band spectra; before, during and after disc-loss.

inner disc appears similar to the rapidly forming inner disc observed in X Per in February 1995 (Tarasov \& Roche 1995).

CGS4 spectra reveal $\mathrm{Br} \gamma$ in absorption (Figure 3), echoing $\mathrm{H} \alpha$; again this is strongly suggestive of general disc loss. However He I $2.06 \mu \mathrm{m}$ (Figure 3) and $1.08 \mu \mathrm{m}$ remain in emission (as are the wings of He I 6678 ). Such a line ratio is very unusual in Be stars. We interpret this as signifying a small dense disc to enable He I emission, whose small extent and large ionization prevents significant Brackett and Balmer emission.

Between 24 April (Optical spectra, Figure 1) and 30 July ( $J H K$ photometry, Figure 2) the disc has returned to it's former emission strength; $\mathrm{K}$ band spectra from 14 August verify this (Figure 3) with resumed $\mathrm{Br} \gamma$ emission.

Previous disc loss events (in X Per, $\gamma$ Cas) have rebuilt fairly continuously over weeks-months. Thus the standstill of between 7-11 months is unusual and demonstrates the episodic nature of disc renewal, as well as the stability of intermediate disc states.

Acknowledgments. We wish to express our gratitude towards the ING service programme, Douglas Gies and Pierre Maxted for acquiring spectra for us, and to Simon Clark and Ignacio Negueruela for helpful suggestions and discussions. NJH is in receipt of a PPARC studentship.

\section{References}

Clark, J.S., Tarasov, A.E., Steele, I.A., Coe, M.J., Roche, P., Shrader, C., Buckley, D.A.H., Larionov, V., Larionova, L., Lyuty, V.M., Zaitseva, G.V., Grunsfeld, J., Fabregat, J., Parise R. 1998, MNRAS 294, 165

Coe, M.J., Carpenter, G.F., Engel, A.R., Quenby J.J. 1975 Nat. 256, 630 Haigh, N.J., Coe, M.J., Steele, I.A., Fabregat, J. 1999, MNRAS, in press Hayakawa, S. 1981 Space Sci.Rev. 29, 22

Rosenberg, F.D., Eyles, C.J., Skinner, C.G., Willmore, A.P. 1975, Nat. 256, 631 Steele, I.A., Negueruela, I., Coe, M.J., Roche, P. 1998, A\&A 297, L5

Tarasov, A.E., Roche, P. 1995, MNRAS 276, L19 